\title{
CORTICAL OBJECT SEGREGATION AND CATEGORIZATION BY MULTI-SCALE LINE AND EDGE CODING
}

\author{
João Rodrigues \\ University of Algarve - Escola Superior de Tecnologia \\ Campus da Penha, 8005-139 Faro, Portugal \\ jrodrig@ualg.pt \\ J.M.Hans du Buf \\ University of Algarve - Vision Laboratory - FCT \\ Campus de Gambelas, 8005-139 Faro, Portugal \\ dubuf@ualg.pt
}

Key words: Visual cortex, line and edge detection, multi-scale, visual reconstruction, recognition, categorization.

\begin{abstract}
In this paper we present an improved scheme for line and edge detection in cortical area V1, based on responses of simple and complex cells, truly multi-scale with no free parameters. We illustrate the multi-scale representation for visual reconstruction, and show how object segregation can be achieved with coarse-to-finescale groupings. A two-level object categorization scenario is tested in which pre-categorization is based on coarse scales only, and final categorization on coarse plus fine scales. Processing schemes are discussed in the framework of a complete cortical architecture.
\end{abstract}

\section{INTRODUCTION}

The visual cortex detects and recognizes objects by means of the "what" and "where" subsystems. The "bandwidth" of these systems is limited: only one object can be attended at any time (Rensink, 2000). In a current model (Deco and Rolls, 2004), the ventral what system receives input from area V1 which proceeds through V2 and $\mathrm{V} 4$ to $\mathrm{IT}^{1}$. The dorsal where system connects V1 and V2 through MT to area PP. Both systems are controlled, top-down, by attention and short-term memory with object representations in PF, i.e. a what component from PF46v to IT and a where component from PF46d to PP. The bottomup (visual input code) and top-down (expected object and position) data streams are necessary for obtaining size, rotation and translation invariance.

Signal propagation from the retinas through the LGN and areas V1, V2 etc., including feature extractions in V1 and groupings in higher areas, takes time. Object recognition is achieved in 150-200 msec, but category-specific activation of PF cortex starts after about $100 \mathrm{msec}$ (Bar, 2004). In addition, IT cortex first receives coarse-scale information and later finescale information. Apparently, one very brief glance is sufficient for the system to develop a gist of the

\footnotetext{
${ }^{1} \mathrm{IT}=$ inferior-temporal cortex, MT = medial temporal, $\mathrm{PP}=$ posterior parietal, $\mathrm{PF}=$ prefrontal, $\mathrm{LGN}=$ lateral geniculate nucleus.
}

contents of an image. This implies that some information propagates very rapidly and directly to "attention" in PF in order to pre-select possible object templates and positions that then propagate down the what and where systems. This process we call object categorization, which cannot be obtained by the CBF model (Riesenhuber and Poggio, 2000) because categorization (e.g. a cat) is obtained by grouping detection cells (cat1, cat2, cat3). In other words, categorization would be obtained after recognition. In contrast, the LF model (Bar, 2004; Oliva et al., 2003) assumes that categorization is obtained before recognition: Low Frequency information that passes directly from V1/V2 to PF, although the LF information actually proposed consists of lowpass-filtered images but not e.g. outputs of simple and complex cells in V1 tuned to low spatial frequencies.

In this paper we present an improved scheme for multi-scale line/edge extraction in $\mathrm{V} 1$, and explore the importance of this multi-scale representation for object segregation and categorization. Since experiments with possible LF models based on lowpassfiltered images_-following (Bar, 2004) — gave rather disappointing results, which is due to smeared blobs of objects that lack any structure, we propose that categorization is based on coarse-scale line/edge representations. The multi-scale keypoint representation also extracted in V1, which was shown to be very important for Focus-of-Attention and e.g. face detection (Rodrigues and du Buf, 2005a; Rodrigues and du Buf, 
2005b), will not be employed here. This means that different models, including simple, complex and end-stopped cells (Heitger et al., 1992), texture inhibition (Grigorescu et al., 2003), keypoint detection (Rodrigues and du Buf, 2004b), line/edge detection (Grigorescu et al., 2003; Rodrigues and du Buf, 2004b; van Deemter and du Buf, 1996; Elder and Zucker, 1998), disparity (Fleet et al., 1991; Rodrigues and du Buf, 2004a), figure-ground segregation (Hupe et al., 2001; Zhaoping, 2003), Focus-of-Attention (Itti and Koch, 2001; Rodrigues and du Buf, 2005b) and face/object recognition (Smeraldi and Bigun, 2002) must be integrated into a multi-feature and multi-layer framework, e.g. (Rasche, 2005; Hubel, 1995).

In Section 2 we present line/edge detection. Section 3 deals with the multi-scale representation, and Section 4 with visual reconstruction. In Section 5 a possible scheme for figure-ground segregation is presented, followed by categorization in Section 6. We conclude with a discussion in Section 7.

\section{LINE AND EDGE DETECTION}

Gabor quadrature filters provide a model of receptive fields (RFs) of cortical simple cells, e.g. (Grigorescu et al., 2003; Rodrigues and du Buf, 2004b).

We apply filters with an aspect ratio of 0.5 and halfresponse width of one octave. The scale $s$ will be given by $\lambda$, the wavelength, in pixels. We can apply a linear scaling with hundreds of quasi-continuous scales, although we will exploit much less scales here.

Responses of even and odd simple cells, which correspond to the real and imaginary parts of a Gabor filter, are denoted by $R_{s, i}^{E}(x, y)$ and $R_{s, i}^{O}(x, y), s$ being the scale, $i$ the orientation $\left(\theta_{i}=i \pi /\left(N_{\theta}-1\right)\right)$ and $N_{\theta}$ the number of orientations (here we use $N_{\theta}=8$ ). Responses of complex cells are modelled by the modulus $C_{s, i}(x, y)=\left[\left\{R_{s, i}^{E}(x, y)\right\}^{2}+\left\{R_{s, i}^{O}(x, y)\right\}^{2}\right]^{1 / 2}$.

A basic scheme for single-scale line and edge detection based on responses of simple cells works as follows (van Deemter and du Buf, 1996): a positive (negative) line is detected where $R^{E}$ shows a local maximum (minimum) and $R^{O}$ shows a zero crossing. In the case of edges the even and odd responses are swapped. This gives 4 possibilities for positive and negative events. An improved scheme (Rodrigues and du Buf, 2004b) consists of combining responses of simple and complex cells, i.e. simple cells serve to detect positions and event types, whereas complex cells are used to increase the confidence. Since the use of Gabor modulus (complex cells) implies a loss of precision at vertices (du Buf, 1993), increased precision was obtained by considering multiple scales (neighboring micro-scales).

The algorithms described above work reasonably well but there remain a few problems: (a) either one scale is used or only a very few scales for increasing confidence, (b) some parameters must be optimized for specific input images or even as a function of scale, (c) detection precision can be improved, and (d) detection continuity at curved lines/edges must be guaranteed.

Here we present an improved algorithm with no free parameters, truly multi-scale, and with new solutions for problems (c) and (d). With respect to precision, simple and complex cells respond beyond line and edge terminations, for example beyond the corners of a rectangle. In addition, at line or edge crossings, detection leads to continuity of the dominant events and gaps in the sub-dominant events. These gaps must be reduced in order to reconstruct continuity. Both problems can be solved by introducing new inhibition schemes, like the radial and tangential ones used in the case of keypoint operators (Rodrigues and du Buf, 2004b). Here we use lateral (L) and crossorientation (C) inhibition, defined as

$$
\begin{gathered}
I_{s, i}^{L}(x, y)=\left[C_{s, i}\left(x+d \mathcal{C}_{s, i}, y+d \mathcal{S}_{s, i}\right)\right. \\
\left.-C_{s, i}\left(x-d \mathcal{C}_{s, i}, y-d \mathcal{S}_{s, i}\right)\right]^{+} \\
+\left[C_{s, i}\left(x-d \mathcal{C}_{s, i}, y-d \mathcal{S}_{s, i}\right)\right. \\
\left.-C_{s, i}\left(x+d \mathcal{C}_{s, i}, y+d \mathcal{S}_{s, i}\right)\right]^{+}
\end{gathered}
$$

and

$$
\begin{gathered}
I_{s, i}^{C}(x, y)=\left[C_{s,\left(i+N_{\theta} / 2\right)}\left(x+2 d \mathcal{C}_{s, i}, y+2 d \mathcal{S}_{s, i}\right)\right. \\
\quad-2 . C_{s, i}(x, y) \\
\left.\quad+C_{s,\left(i+N_{\theta} / 2\right)}\left(x-2 d \mathcal{C}_{s, i}, y-2 d \mathcal{S}_{s, i}\right)\right]^{+},
\end{gathered}
$$

where $[\cdot]^{+}$denotes suppression of negative values, $\left(i+N_{\theta} / 2\right) \perp i, \quad \mathcal{C}_{s, i}=\cos \theta_{i}, \quad \mathcal{S}_{s, i}=\sin \theta_{i}$ and $d=0.6 \mathrm{~s}$. Inhibition is applied to complex cell responses, where $\beta$ controls the strength of the inhibition (we use $\beta=1.0$ ), i.e. $\hat{C}_{s, i}=$ $\left[C_{s, i}(x, y)-\beta\left(I_{s, i}^{L}(x, y)+I_{s, i}^{C}(x, y)\right)\right]$.

Line/edge detection is achieved by constructing a few cell layers on top of simple and complex cells. The first layer serves to select active regions and dominant orientations. At each position, responses of complex cells are summed $\left(\hat{C}_{s}=\sum_{i=0}^{N_{\theta}-1} \hat{C}_{s, i}\right)$, and at positions where $\hat{C}_{s}>0$ an output cell is activated. At active output cells, the dominant orientation is selected by gating one complex cell on the basis of nonmaximum suppression of $\hat{C}_{s, i}$. The gating is confirmed or corrected by excitation/inhibition of dominant orientations in a local neighborhood.

In the second layer, event type and position are determined on the basis of active output cells (1st layer) and gated simple and complex cells. A first cell complex checks simple cells $R_{s, i}^{E}$ and $R_{s, i}^{O}$ for a local maximum (or minimum by rectification) using a dendritic 
field size of $\pm \lambda / 4, \lambda$ being the wavelength of the simple cells (Gabor filter). The active output cell is inhibited if there is no maximum or minimum. A second cell complex does exactly the same on the basis of complex cells. A third cell complex gates four types of zero-crossing cells on the basis of simple cells, again on $\pm \lambda / 4$. If there is no zero-crossing, the output cell is inhibited. If there is a zero-crossing, the active output cell at the position of the zero-crossing cell determines event position and the active zero-crossing cell determines event type.

In the third layer, the small loss of accuracy due to the use of complex cells in the second layer is compensated. This is done by correcting local event continuity, considering the information available in the second layer, but by using excitation of output cells by means of grouping cells that combine simple and complex cells tuned to the same and two neighboring orientations. The latter process is an extension of linear grouping (van Deemter and du Buf, 1996) and a simplification of using banana wavelets (Krüger and Peters, 1997). In the same layer event type is corrected in small neighborhoods, restoring type continuity, because the cell responses may be distorted by interference effects when two events are very close (du Buf, 1993).

Figure 1 shows three input images together with fine-scale detection results in which positive and negative lines and edges are coded by different gray levels. Detection accuracy is very good and there are many small events due to low-contrast texturesFig. 1 does not show event amplitudes-and the fact that there is no threshold value in the detection scheme. For comparing results with those obtained by standard, edge-only (!) detection algorithms we refer to (Heath et al., 2000) and

http://marathon.csee.usfledge/edge_detection.html

\section{MULTIPLE SCALES}

For illustrating scale space we can create an almost continuous, linear scaling with hundreds of scales on $\lambda=[4,52]$, but here we will present only a few scales to show complications. Figure 2 shows events detected at five scales in the case of ideal, solid square and star objects. At fine scales (at the left) the edges of the square are detected, as are most parts of the star, but not at the very tips of the star. This illustrates an important difference between normal computer vision and developing cortical models. The latter must be able to construct brightness maps, and at the tips of the star, where two edges converge, there are very fine lines. The same effect occurs at coarser scales, until entire triangles are detected as lines and even pairs of opposite triangles (at the right). In the case of the
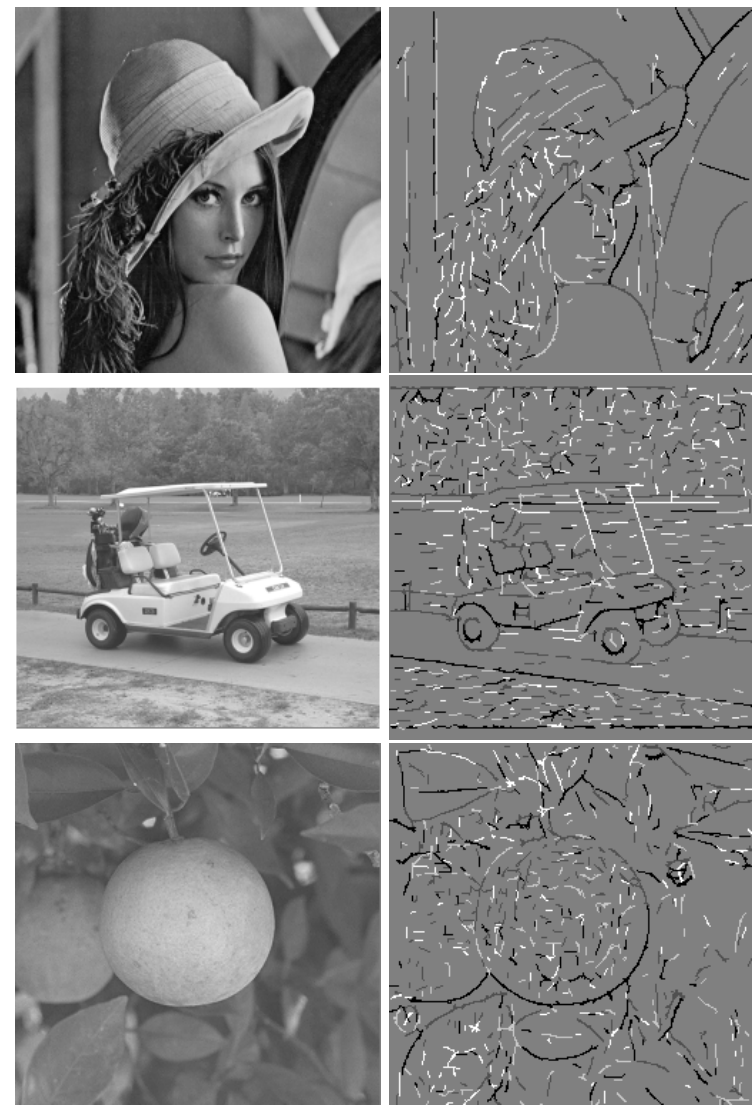

Figure 1: Fine-scale line/edge detection.

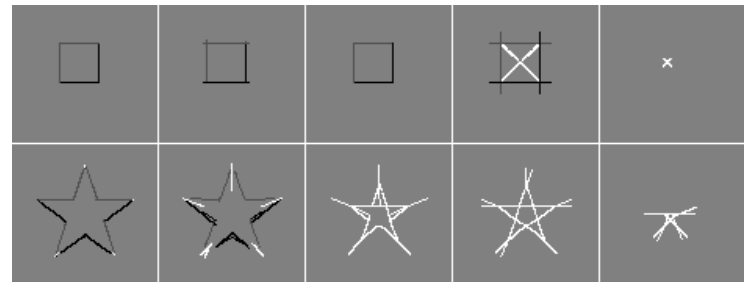

Figure 2: Multi-scale representation of a square and a star, left to right $\lambda=\{4,12,18,24,40\}$.

square, lines will be detected at diagonals, which vanish, also with small amplitudes, at very coarse scales.

Figure 3 shows, left to right, multi-scale event detection in the case of a leaf with, top to bottom, different criteria for scale stability: single scale (no stability check), micro-scale stability (Rodrigues and du Buf, 2004b) over a few neighboring scales, and stability over 10 and 40 scales. This illustrates that detected lines and edges are stable over many scales, which is very important for tasks like visual reconstruction and object recognition. 


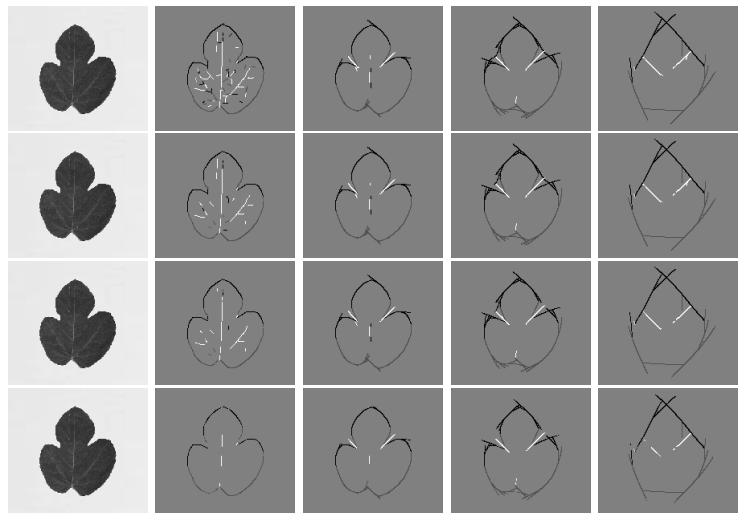

Figure 3: Left to right: original and multi-scale event detection ( $\lambda=\{4,9,16,36\})$; top to bottom: single scale, micro-scale stability, and stability over 10 and 40 scales.

\section{VISUAL RECONSTRUCTION}

Image reconstruction can be obtained by assuming one lowpass filter plus a complete set of (Gabor) wavelets that cover the entire frequency domain - this concept is exploited in image coding. The goal of our visual system is to detect objects, with no need, nor capacity, to reconstruct a complete image of our visual environment, see change blindness and the limited "bandwidth" of the what and where subsystems (Rensink, 2000). Yet, the image that we perceive in terms of brightness must somehow be created. A normal image coding scheme, for example by summing responses of simple cells, requires accumulation in one cell layer which contains a brightness map, but this would require yet another "observer" of this map in our brain. A solution for this dilemma is to assume that detected lines and edges are interpreted symbolically: an active "line cell" is interpreted as a Gaussian intensity profile with a certain orientation, amplitude and scale, the size of the profile being coupled to the scale of the underlying simple and complex cells. An active "edge cell" is interpreted the same way, but with a bipolar, Gaussian-truncated, error-function profile. As for image coding, this representation must be complemented with a lowpass filter, a process that happens to exist by means of retinal ganglion cells with photoreceptive dendritic fields not (in)directly connected to rods and cones (Berson, 2003).

One brightness model (du Buf, 1994) is based on the symbolic line/edge interpretation. It explains Mach bands (Pessoa, 1996) by the fact that responses of simple cells do not allow to distinguish between lines and ramp edges, and it was shown to be able to predict many brightness illusions such as simultaneous brightness contrast and assimilation, which are two opposite induction effects (du Buf and Fischer,

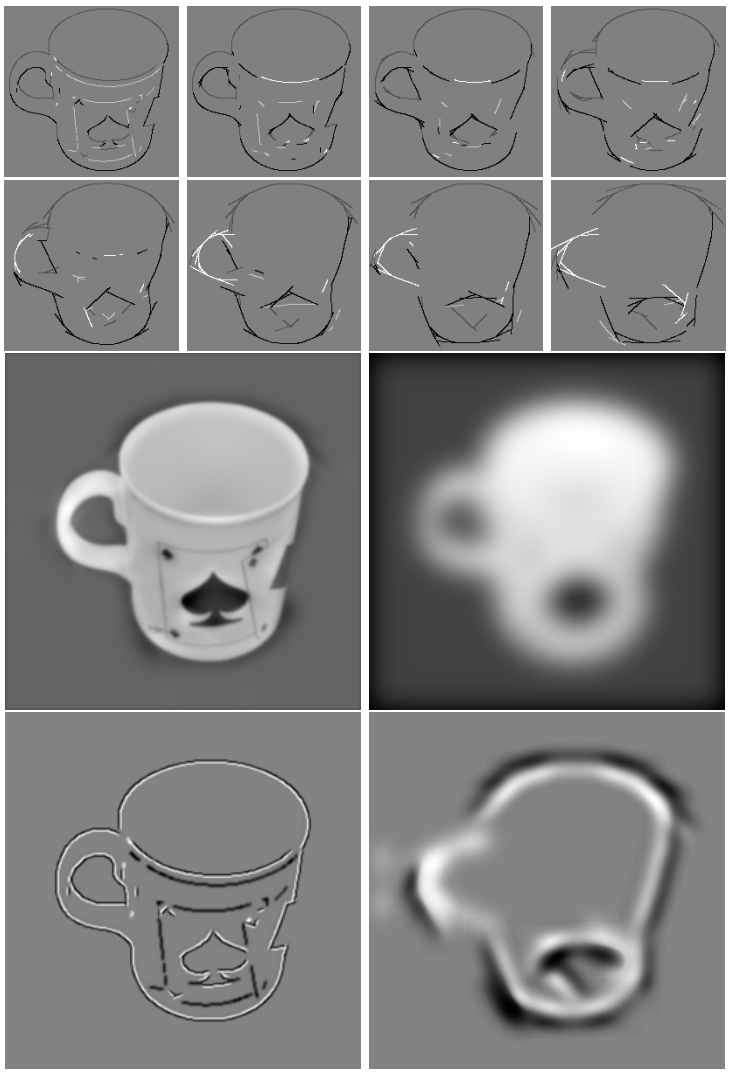

Figure 4: Multi-scale representation of a mug (top). Reconstruction (middle-left) by combining lowpass (middle-right) and line/edge interpretations (bottom).

1995). Here we will not go into more detail; we will only illustrate the symbolic reconstruction process in 2D (the model referred to above was tested in 1D).

Figure 4 (top) shows the multi-scale line/edge representation in the case of a mug that will be used in categorization (Fig. 7 shows the original image). Figure 4 also illustrates visual reconstruction based on a lowpass-filtered image (middle-right), symbolic line/edge interpretations at six scales, two of which are shown (bottom), and the combination (middleleft). The fuzzy contour of the lowpass image is corrected by adding the line/edge interpretations. Using more scales leads to better reconstructions, but the relative weighting of the lowpass and scale components requires further investigation.

\section{OBJECT SEGREGATION}

Until here we have illustrated multi-scale line/edge detection in area V1 and the symbolic interpretation for reconstruction, but the goal of the visual cor- 
tex is to detect and recognize objects by means of the what and where systems. An essential step, related to object categorization, is figure-ground segregation. Figures 2, 3 and 4 show typical event maps of different objects, with detail at fine scales and reduced, "sketchy" information at coarse scales. At a coarse level, each individual event (group of responding line/edge cells) or connected group of events corresponds to one object. Each event at a coarse scale is related to events at one finer scale, which can be slightly displaced or rotated. This relation is modeled by downprojection using grouping cells with a dendritic field, the size of which defines the region of influence. Responding event cells at all scales activate grouping cells, which yields regions of influence (Fig. 5 middle-left). This coarse-to-fine-scale process is complemented by inhibition: other grouping cells at the finest scale are activated by responding event cells at that scale and these grouping cells excitate the grouping cells at the one coarser scale but inhibit active grouping cells outwards, shown red in Fig. 5 (bottom). This results in a figure-ground map at the first coarser scale "above" the finest scale (Fig. 5 top-right). Results shown were obtained with $\lambda=[4,52], \Delta \lambda=4$.

A process in $\mathrm{V} 1$ as described above can be part of the where system, but it needs to be embedded into a complete architecture (Deco and Rolls, 2004). In addition, when two objects are very close, they will become connected at coarse scales and separation is only possible by the what system that checks features (lines, edges, keypoints) of individual objects at finer scales. In other words, object segregation is likely to be driven by "attention" in PF cortex, for example by means of templates that consist of coarse-scale line/edge representations, and this process is related to object categorization.

\section{OBJECT CATEGORIZATION}

Object recognition is a clearly defined task: a certain cat, like the neighbors' red tabby called Toby, is recognized or not. Categorization is more difficult to define because there are different levels, for example (a) an animal, (b) one with four legs, (c) a cat, and (d) a red tabby, before deciding between our own red tabby called Tom and his brother Toby living next door. It is as if we were developing categorization by very young children: once they are familiar with the family's cat, every moving object with four legs will be a cat. With age, more features will be added. Here we explain our experiments with a twolevel approach; three types of objects (horses, cows, dogs) are first grouped (animal), which we call precategorization, after which categorization determines

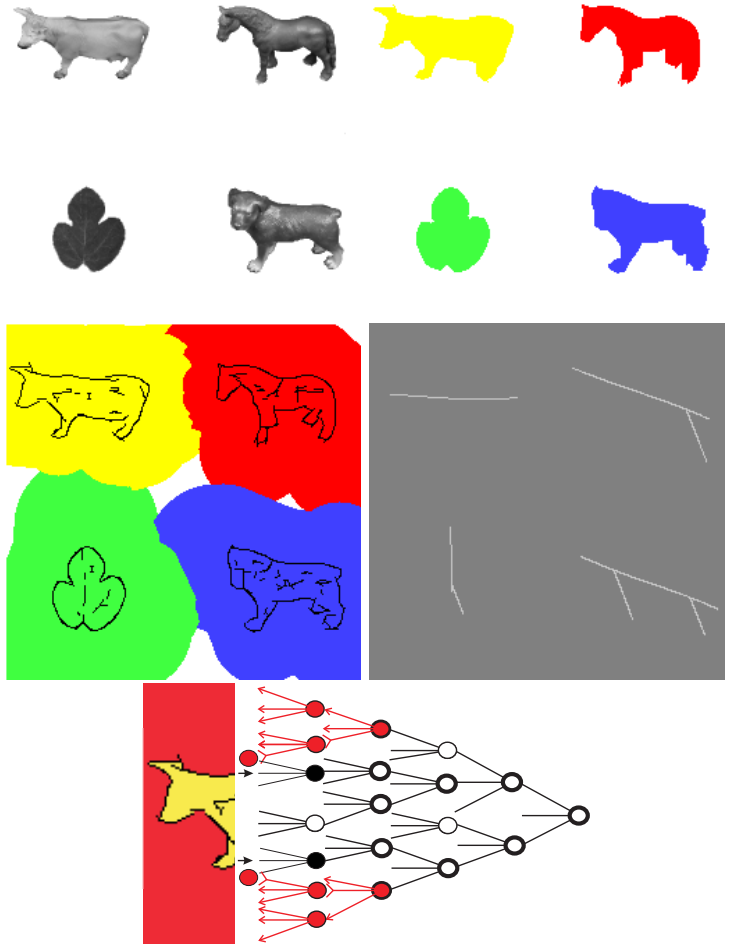

Figure 5: Input image with four objects (top-left), coarsescale representation (middle-right; $\lambda=52$ ), regions of influence (middle-left), final segregation (top-right), and activation/inhibition of grouping cells (bottom).

the type of animal. Instead of creating group templates in memory on the basis of lowpass-filtered images as proposed by the LF model (Bar, 2004; Oliva et al., 2003), we will exploit coarse-scale line and edge templates. In addition, pre-categorization will be based on line/edge templates of contours, i.e. solid objects, available through segregation (Fig. 5), to generalize shape and to eliminate surface detail.

We used the ETH-80 database (Leibe and Schiele, 2003), in which all images are cropped such that they contain only one object, centered, against a $20 \%$ background. Images were rescaled to a size of $256 \times 256$ pixels. We selected 10 different images of 8 groups (dogs, horses, cows, apples, pears, tomatos, cups/mugs and cars), in total 80 images. Figure 7 shows examples. Because views of objects are also normalized (e.g. all animals with the head to the left), and because different objects within each group are characterized by about the same line/edge representations at coarser scales, group templates can be constructed by combining randomly selected images. The multi-scale line/edge representation was computed at 8 scales equally spaced on $\lambda=[4,32]$. 


\section{Pre-categorization}

Here the goal is to select one of the groups: animal, fruit, cup or car. We used the three coarsest scales with $\lambda$ equal to 24,28 and 32 pixels. Group templates were created by combining all images (30 animals, 30 fruits, 10 cups, 10 cars), and by random selections of half (15 and 5) and one third (10 and 3) of all images. By using more images, a better generalization can be obtained, for example the legs of animals can be straight down or more to the front (left). Figure 6 shows examples of segregated objects and line/edge templates when using half of all images. For each group template, at each of the three scales, a positional relaxation area was created around each responding event cell, by assuming grouping cells with a dentritic field size coupled to the size of underlying complex cells (Bar, 2003). These grouping cells sum the occurence of events in the input images around event positions in the templates, a sort of local correlation, and activities of all grouping cells were then grouped together (global correlation). The final groupings were compared over the 4 templates, scale by scale, and the template with maximum response was selected. Finally, the template with the maximum number of correspondences over the 3 scales was selected. The following table summarizes results (misclassified images) in the form mean(st. deviation):

\begin{tabular}{l|rrr} 
template & all & half & third \\
construction & $30 / 10$ & $15 / 5$ & $10 / 3$ \\
\hline no relaxation & 0 & $5.7(0.6)$ & $8.0(1.7)$ \\
with relaxation & 0 & $3.0(1.0)$ & $4.3(0.6)$
\end{tabular}

Obviously, positional relaxation leads to better results when not all images are used in building the templates, and using more images is always better. Using relaxation and more images increases shape generalization, however with the risc of running into over-generalization, which did not occur in our tests. On the average, different random selections gave very similar results when the three sub-groups (horses/cows/dogs and apples/pears/tomatos) were about equally represented. Most errors occurred, with and without relaxation, between car/animal and cup/fruit.

\section{Categorization}

After pre-categorization, assuming zero errors, there remains one problem in our test scenario: the animal group must be separated into horse, cow and $\mathrm{dog}$, and the fruit group into apple, pear and tomato. We could have used 6 templates (cups and cars have already been categorized), but we experimented with 8 templates and all 80 images, and applied the multi-scale line/edge representations at all 8 scales
( $\lambda$ equal to 4, 8, 12, 16, 20, 24, 28 and 32) of the real input images (not solid objects). We did this because categorization is supposed to be done after precategorization, i.e., when also fine-scale information has propagated to IT cortex (see Introduction).

Templates were constructed as above with random selections. Final groupings (global correlation) were compared over the 8 scales and the one with most coherent (maximum) correspondences was selected (in the case of 4-4 we simply took the last one). The following table presents results (misclassifications) obtained with positional relaxation:

\begin{tabular}{l|rrr} 
template & all & half & third \\
construction & 10 & 5 & 3 \\
\hline errors & 0 & $9.3(2.1)$ & $12.7(4.0)$
\end{tabular}

Again, by using more images in building the templates, generalization is improved and the number of mis-categorized images decreases. When using half (5) or even one third (3) of all images, all car and cup images were correctly categorized, and no fruits were categorized as animals and vice versa. Typical miscategorizations were dog/cow, horse/dog, horse/cow and apple/tomato. Figure 7 shows, apart from examples of images and group templates created by combining 5 images (top), more difficult images with a white triangle in the bottom-right corner. It should be stressed that this is an extremely difficult test, because no color information has been used and apples and tomatos have the same, round shape. By contrast, all pear images, with a tapered shape, have been correctly categorized. The fact that most problems occurred with the animals was expected, given the minute differences of heads, necks and tails (Fig. 7). Categorization is the final step before recognition in which attention shifts to finer scales that reflect minute differences. Nevertheless, only about 9 errors in 80 images (the 50/50 "training and testing" scenario) is a very promising starting point for refining the algorithms, for example by using a more hierarchical scenario with more categorization steps, in which attention is systematically steered from coarse to fine scales.

\section{DISCUSSION}

Computer vision for realtime applications requires tremendous computational power because all images must be processed from the first to the last pixel. Probing specific objects on the basis of already acquired context may lead to a significant reduction of processing. This idea is based on a few concepts from our visual cortex (Rensink, 2000): (1) our physical surround can be seen as memory, i.e. there is no need to construct detailed and complete maps, (2) the bandwidth of the what and where systems is limited, 


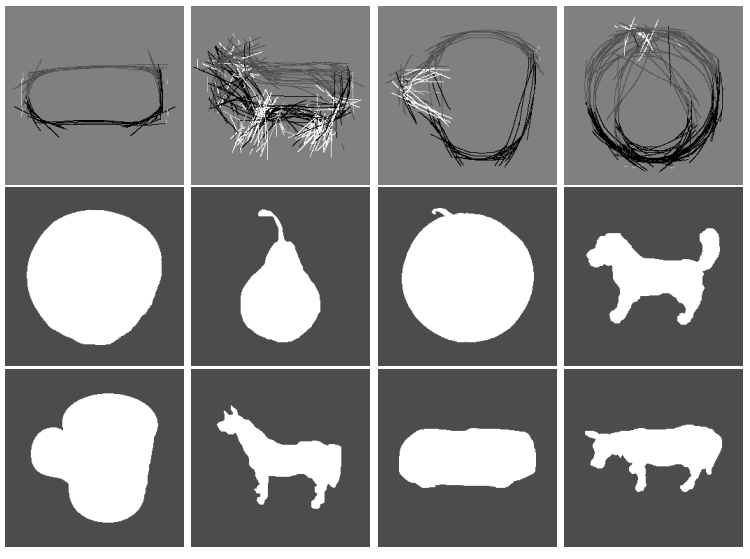

Figure 6: Top: templates for pre-categorization based on 15 and 5 images at $\lambda=32$. Bottom: examples of segregated objects.

i.e. only one object can be probed at any time, and (3) bottom-up, low-level feature extraction is complemented by top-down hypothesis testing, i.e. there is a rapid convergence of activities in dendritic/axonal cell connections from V1 to PF cortex.

In previous papers we have shown that keypoint scale-space is ideal for constructing saliency maps for Focus-of-Attention (FoA) (Rodrigues and du Buf, 2005a), and that faces can be detected by grouping facial landmarks defined by keypoints at eyes, nose and mouth (Rodrigues and du Buf, 2005b). On the other hand, line/edge scale-space may be ideal for object and face recognition. Obviously, these two representations in V1 complement each other and both can be used for object detection, categorization and recognition. Our impression is that keypoints are used more in the fast where system (FoA), whereas lines and edges are exploited more in the slower what system. However, this still needs to be tested in the context of a complete cortical architecture with ventral and dorsal data streams that link V1 to attention in PF cortex (Deco and Rolls, 2004).

In this paper we presented an improved scheme for line and edge detection in V1, and illustrated the multi-scale representation for visual reconstruction. This representation, in combination with a lowpass filter, yields a reconstruction that is suitable for extending our brightness model (du Buf and Fischer, 1995) from 1D to 2D, for example for modelling brightness illusions.

We also presented a plausible scheme for object segregation, which results in binary, solid objects that can be used to obtain a rapid pre-categorization on the basis of coarse-scale information only. This approach works much better if compared to using lowpass-filtered images, i.e., smeared blobs that lack

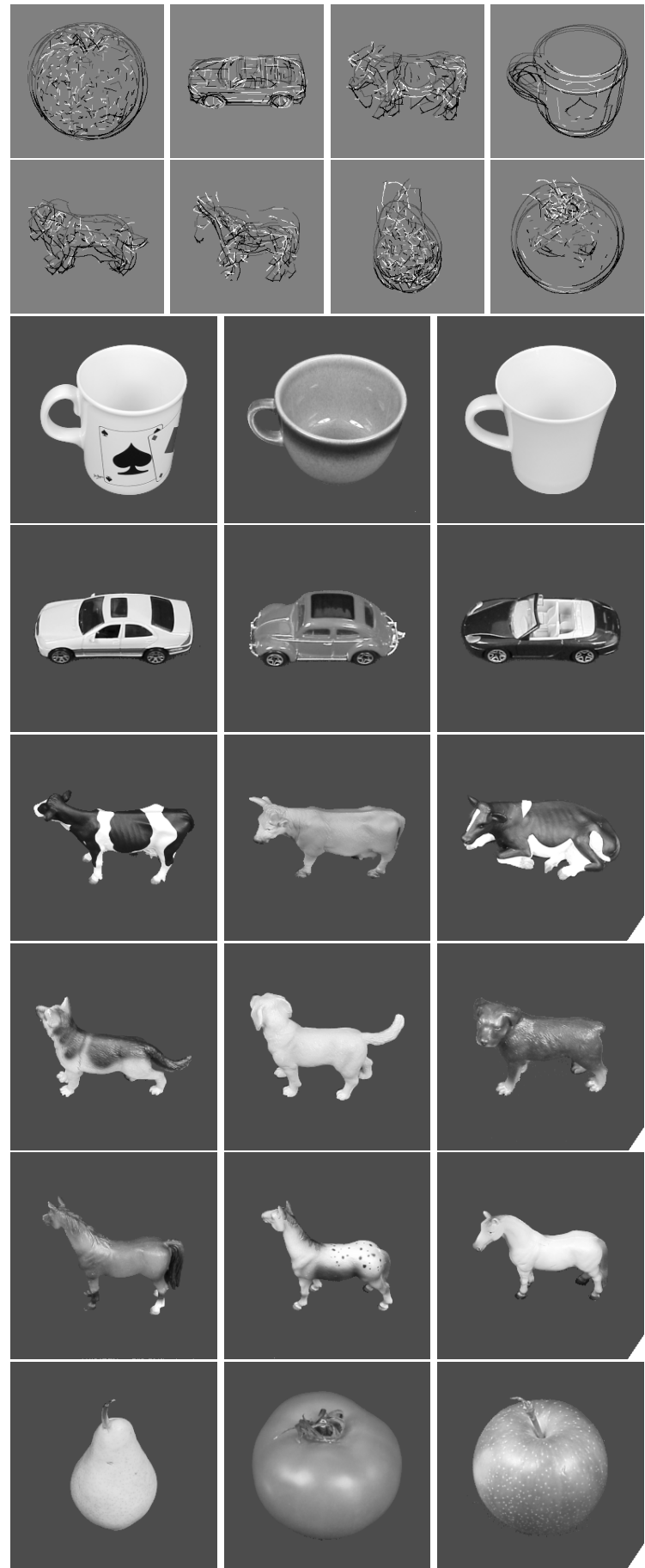

Figure 7: Top: templates for final categorization based on 5 images at $\lambda=8$. Bottom: examples of object images, the more difficult ones with a white triangle in the bottom-right corner.

object-specific characteristics (Bar, 2004; Oliva et al., 2003). Final categorization was tested by using real 
objects and more scales, coarse and fine. The results obtained are very promising, taking into account that the tested schemes are extremely simple. Only a fraction of available information, i.e., the line/edge code without amplitude and color information, and without a linking of scales as explored in the segregation model, has been used so far. More extensive tests are being conducted, with more images and objects, concentrating on a linking of scales and a steering of attention from coarse to fine scales. Such improved schemes are expected to yield better results, from very fast detection (where) to slower categorization (where/what) to recognition (what). The balance between keypoint and line/edge representations is an important aspect.

Acknowledgements: This research is partly financed by PRODEP III Medida 5, Action 5.3, and by the FCT program POSI, framework QCA III.

\section{REFERENCES}

Bar, M. (2003). A cortical mechanism for triggering topdown facilitation in visual object recognition. J. Cogn. Neuroscience, (15):600-609.

Bar, M. (2004). Visual objects in context. Nature Reviews: Neuroscience, 5:619-629.

Berson, D. (2003). Strange vision: ganglion cells as circadian photoreceptors. TRENDS in Neurosciences, 26(6):314-320.

Deco, G. and Rolls, E. (2004). A neurodynamical cortical model of visual attention and invariant object recognition. Vision Res., (44):621-642.

du Buf, J. (1993). Responses of simple cells: events, interferences, and ambiguities. Biol. Cybern., 68:321-333.

du Buf, J. (1994). Ramp edges, Mach bands, and the functional significance of simple cell assembly. Biol. $C y$ bern., 70:449-461.

du Buf, J. and Fischer, S. (1995). Modeling brightness perception and syntactical image coding. Optical Eng., 34(7):1900-1911.

Elder, J. and Zucker, S. (1998). Local scale control for edge detection and blur estimation. IEEE Tr. PAMI, 20(7):699-716.

Fleet, D., Jepson, A., and Jenkin, M. (1991). Phase-based disparity measurement. CVGIP: Image Understanding, 53(2):198-210.

Grigorescu, C., Petkov, N., and Westenberg, M. (2003). Contour detection based on nonclassical receptive field inhibition. IEEE Tr. IP, 12(7):729-739.

Heath, M., Sarkar, S., Sanocki, T., and Bowyer, K. (2000). A robust visual method for assessing the relative performance of edge-detection algorithms. IEEE $\mathrm{Tr}$. PAMI, 19(12):1338-1359.
Heitger, F., Rosenthaler, L., von der Heydt, R., Peterhans, E., and Kubler, O. (1992). Simulation of neural contour mechanisms: from simple to end-stopped cells. Vision Res., 32(5):963-981.

Hubel, D. (1995). Eye, brain and vision. Scientific American Library.

Hupe, J., James, A., and Bullier, J. (2001). Feedback connections act on the early part of the responses in monkey visual cortex. J. Neurophysiol., 85(1):134-144.

Itti, L. and Koch, C. (2001). Computational modeling of visual attention. Nature Reviews: Neuroscience, 2(3):194-203.

Krüger, N. and Peters, G. (1997). Object recognition with banana wavelets. Proc. 5th Europ. Symp. Artificial Neural Networks, pages 61-66.

Leibe, B. and Schiele, B. (2003). Analyzing appearance and contour based methods for object categorization. Proc. Int. Conf. Comp. Vision Pattern Recogn, Madison (Wisconsin), pages 409-415.

Oliva, A., Torralba, A., Castelhano, M., and Henderson, J. (2003). Top-down control of visual attention in object detection. Int. Conf. Im. Proc., 1:253-256.

Pessoa, L. (1996). Mach bands: how many models are possible? Recent experimental findings and modeling attemps. Vision Res., 36:3205-3227.

Rasche, C. (2005). The making of a neuromorphic visual system. Springer.

Rensink, R. (2000). The dynamic representation of scenes. Visual Cogn., 7(1-3):17-42.

Riesenhuber, M. and Poggio, T. (2000). Cbf: A new framework for object categorization in cortex. 1st IEEE Int. Worksh. Biologically Motivated Computer Vision, Seoul (Korea), pages 1-9.

Rodrigues, J. and du Buf, J. (2004a). Vision frontend with a new disparity model. Early Cogn. Vision Worksh., Isle of Skye (Scotland)

www.cn.stir.ac.uk/ecovision-ws/.

Rodrigues, J. and du Buf, J. (2004b). Visual cortex frontend: integrating lines, edges, keypoints and disparity. Proc. Int. Conf. Image Anal. Recogn., Springer LNCS 3211(1):664-671.

Rodrigues, J. and du Buf, J. (2005a). Multi-scale cortical keypoint representation for attention and object detection. 2nd Iberian Conf. on Patt. Recogn. and Image Anal., Springer LNCS 3523:255-262.

Rodrigues, J. and du Buf, J. (2005b). Multi-scale keypoints in V1 and face detection. 1st Int. Symp. Brain, Vision and Artif. Intell., Naples (Italy), Springer LNCS 3704:205-214.

Smeraldi, F. and Bigun, J. (2002). Retinal vision applied to facial features detection and face authentication. Pattern Recogn. Letters, 23:463-475.

van Deemter, J. and du Buf, J. (1996). Simultaneous detection of lines and edges using compound Gabor filters. Int. J. Patt. Recogn. Artif. Intell., 14(6):757-777.

Zhaoping, L. (2003). V1 mechanisms and some figureground and border effects. J. Physiology, 97:503-515. 